\title{
Indigenous Tweeting for Language Survival: The Māori-Language Profile
}

\author{
Paora Mato, Te Taka Keegan \\ Faculty of Computing and Mathematical Sciences \\ University of Waikato, New Zealand
}

\begin{abstract}
Indigenous and minority language groups are increasingly embracing the power of web-based technology as they struggle to ensure the continued health and survival of their own languages. Māori, the indigenous race of New Zealand, are no exception. Initiatives involving the Microsoft Corporation, Moodle and Google Inc. have resulted in a range of localized interfaces now available in the Mãori language. More recently, the Mãori language has been made available on mobile devices, physical self-service machines and social media technology.
\end{abstract}

This research investigates the development of online Māori-language communities using Twitter. The investigation was made possible by the information capture and trending functionality of the Indigenous Tweets website. It is evident that the development of vibrant online indigenous and minority language communities is underpinned by the ease in which the speakers are able to find each other amidst the sheer volume of global languages that dominate social media. It is also evident for te reo Māori that the bulk of Māori-language tweets arise from initiatives that seek to disseminate translated information rather than illicit interaction from other users. Whether or not this could be deemed suitable for developing online language communities is yet to be determined. However, some individuals were identified who were focused on engaging users in online conversations in te reo Māori.

\section{Introduction}

Te reo Māori, the indigenous language of Aotearoa/New Zealand, is one of many at-risk indigenous languages. Various initiatives over the past 40 years have sought to halt the decline of te reo Māori and increase the number of fluent speakers. While early language initiatives were created that focused on education and community language development [1] more recently the use of technology is making significant inroads into the areas of language acquisition, wider communication and networking. As schools are increasingly using this technology in their education activities, indigenous language schools are making initial attempts to follow suit.
Recent technologies have included localized interfaces for a selection of computer applications, mobile technology, physical self-service machines and social media. The use of the Internet is even more significant since these technologies can be more widely dispersed and continuously accessible. In terms of language survival then, one would expect that the ability to develop far-reaching capability, available in at-risk languages, would be advantageous in some manner. Intuitively, the existence of such tools in a global, unbridled environment would offer a variety of options to a language strategy aimed at survival and regeneration. It should also be apparent that the availability of these tailored resources should be complemented with strategies that encourage their extended use in the target language.

'Indigenous' websites already exist that represent indigenous organizations, promote indigenous ecommerce and further the unique concerns of indigenous communities including cultural maintenance and revitalization [2]. Social networking platforms such as Facebook and Twitter have become a way for indigenous people all around the world to connect with others from the same language group regardless of geographic distances.

A recent linguistic survey has identified 500 languages being used on Twitter that include Gamilaraay, an Australian indigenous language with three remaining speakers [3]. Further surveys have identified the increased use of social networking sites such as Facebook and Twitter by students who say they feel comfortable contacting each other to discuss homework, assignments and exams. Sites such as these have allowed people to connect online and use their languages in a natural way [4]. They are also seen as effectively engaging young people who are often considered to be crucial to language revitalization efforts $[1,2,4,5]$.

\section{Research objectives}

Long-term, this research aims to determine the impact of micro-blogging in Twitter on the use of Māori language online. In the short-term, this research has investigated the extent that te reo Māori is being used in this medium. It seems clear that the continued health of te reo Māori is underpinned by the use of the language in as many forums as possible. The development of a vibrant online community, sharing information and ideas using te 
reo Māori, supports the notion of increased language use.

Following this determination, further research would seek to quantify support for creating larger online communities of Māori-language tweeters and to source strategies for how this could and should be done.

\subsection{The Indigenous Tweets Website}

The Indigenous Tweets website (http://indigenoustweets.com/) was developed by Professor Kevin Scannell of the Department of Mathematics and Computer Science at the University of Saint Louis, Missouri, USA. Scannell [6] cites the primary aim of this website is to "help build online language communities through Twitter (p. 1)", hoping that the site will enable speakers of indigenous and minority languages to connect in their own languages amidst "a vast sea of English, French, Spanish and other global languages that dominate Twitter (p. 1)". Indigenous Tweets collates the occurrence of tweets (the postings of micro-blogs using Twitter) in selected minority languages. The website, launched in March 2011, catalogued tweets from 35 chosen languages. By May 2011 the number of indigenous languages identified had more than doubled with tweeter profiles for over 80 languages displayed on the website. User profiles are currently (September 2013) listed for 150 indigenous languages.

Indigenous Tweets identifies languages by searching Twitter for clusters of words it recognises from a database. The database contains text for approximately 500 languages and was compiled using a web crawler to scan blogs, news articles and webpages [7]. Scannell uses tweet data to improve language dictionaries and spell checkers, especially for the Irish language, and says " ... it's cool seeing the language changing day by day, week by week"[8]. This data is used by a program that randomly scans Twitter users and applies a method of statistical language recognition using tri-grams to ascertain what fraction of their tweets contain the target language [3]. Language recognition is based on the analysis of three character sequences ("3grams" or "tri-grams) and "it turns out that computing the statistics of 3 -grams in a given language provides a "fingerprint" that can be used for language identification and a number of other applications" [9]. When a tweet contains a prescribed combination of word sequences, it is captured by Indigenous Tweets as belonging to a specific language. The user's profile is then added to the Indigenous Tweets website and their followers' profiles are also added to a queue for scanning. Profiles are established of tweeters using the various selected mother tongues, enabling speakers using the same languages to get in touch with each other [10].
In collaboration with language groups globally, Scannell is using the data he has gathered from webbased text, written in indigenous and minority languages, to develop basic resources that will help these groups use their language online. "The site's very simple," says Scannell, "you find your language, you click on it, and it takes you to a table of all the people that are tweeting in your language" [10]. He says this work underlies the Indigenous Tweets and Indigenous Blogs projects "which aim to strengthen languages through social media" [9].

\subsection{Indigenous Tweets Language Statistics}

The Indigenous Tweets website currently lists profile information for 150 languages. The top 10 languages recorded by this website, when ranked by the number of user-profiles associated with that language, are shown in Table 1. Note, the statistics displayed in this table and used throughout the paper were retrieved from the Indigenous Tweets website on the $5^{\text {th }}$ September, 2013.

Table 1: Indigenous Tweets Total Numbers

\begin{tabular}{|l|r|r|r|}
\hline Language & Users & Tweets & $\begin{array}{r}\text { Tweets } \\
\text { per user }\end{array}$ \\
\hline Euskara & 17042 & 4629179 & 272 \\
\hline Kreyòl Aylsyen & 14267 & 3801664 & 266 \\
\hline Cymraeg & 14218 & 2461608 & 173 \\
\hline Gaeilge & 7388 & 472825 & 1132 \\
\hline Frysk & 2048 & 571851 & 279 \\
\hline Kapampangan & 1379 & 1560984 & 1798 \\
\hline Asturianu & 749 & 254257 & 64 \\
\hline Gàidhlig & 652 & 93423 & 339 \\
\hline Soomaaliga & 558 & 141671 & 788 \\
\hline Māori & 335 & 89391 & 254 \\
\hline
\end{tabular}

Euskara, with the most number of users, refers to the language spoken by the Basque who are located on both sides of the border of southern France and northern Spain. The language with the second most users is Haitian Creole, a creole language spoken by about 8.5 million people in the Republic of Haiti in the Caribbean. There are a further 3.5 million speakers in a number of countries that include Canada, the USA, France, Cuba, and other Caribbean countries [11]. For the promoters of an online Euskara language community this data indicates some early success. Comparisons between Euskara and Haitian Creole highlights the importance of online language communities as a tool for language health. More users engage in Euskara than Hatian Creole, however, at approximately 12 million 
speakers, Haitian Creole boasts over 12 times more native language speakers than Euskara. Slightly less than $2 \%$ of the Euskara-speaking population are tweeting in their language compared with $0.12 \%$ of the speakers of Haitian Creole tweeting in their own language. As a further comparison, over $2.5 \%$ of the more than half a million [12] speakers of Cymraeg (Welsh) are engaged online within this medium, suggesting some gains for promoters of the Welsh language as well.

The other languages with more than one thousand user profiles are Gaeilge (Irish Gaelic), Frysk (Friesian - Netherlands) and Kapampangan from the Philippines. Te reo Māori (the Māori language) features $10^{\text {th }}$ on the list with 335 user profiles who tweet in te reo Māori.

\subsection{Indigenous Tweets Top User Statistics}

Analysing the statistics of top users is important because it can indicate whether a language community has many users tweeting in an indigenous language or it has few users who are tweeting often in an indigenous language. The top users' tweets have been calculated as a percentage of the total tweets for that language and are displayed in Table 2.

Table 2: Most Indigenous Tweets by single User

\begin{tabular}{|l|l|r|r|}
\hline Language & Top User & Tweets & $\begin{array}{c}\% \text { of } \\
\text { Total }\end{array}$ \\
\hline Euskara & berria & 43464 & $0.9 \%$ \\
\hline Kreyòl Aylsyen & RoodHT509 & 49006 & $1.3 \%$ \\
\hline Cymraeg & newyddcymraeg & 57703 & $2.3 \%$ \\
\hline Gaeilge & aonghusoha & 24336 & $5.2 \%$ \\
\hline Frysk & omropfytsban & 61042 & $10.7 \%$ \\
\hline Kapampangan & itsmeshellie & 19449 & $1.3 \%$ \\
\hline Asturianu & iyangc & 16439 & $6.5 \%$ \\
\hline Gàidhlig & sconewt & 27303 & $29.2 \%$ \\
\hline Soomaaliga & Weedhsan & 15895 & $11.2 \%$ \\
\hline Māori & maonewt & 29810 & $33.4 \%$ \\
\hline
\end{tabular}

Tweeters identified as using Euskara (Basque) generate the highest number of tweets captured by the Indigenous Tweets website. The Euskara user with the highest number of tweets represents slightly less than $1 \%$ of the Euskara total. Top users in other languages, namely Kapampangan (Philippines), Kreyòl Aylsyen (Haitian Creole) and Cymraeg (Welsh) also show relatively low percentages of tweets in their respective languages. This suggests a language community with many tweeters who are tweeting in their indigenous language.
In contrast, a single tweeter in Māori is responsible for $33 \%$ of their language tweets and a single user in Gàidhlig (Scottish Gaelic) constitutes $29 \%$ of their total language tweets. This suggests language communities that have only a few individuals who are tweeting extensively in their language.

\section{Te reo Māori Tweeting}

The statistics posted on the Indigenous Tweets website were examined in further detail in an effort to determine reasons and motivations for tweeting in te reo Māori. It was noted that the top five users provide approximately $80 \%$ of the tweets in Māori while the top 20 users account for almost $90 \%$ of all tweets. In comparison the top 20 Euskara users account for slightly less than $8 \%$ of the total Euskara tweets.

\subsection{Māori Tweets Top User Statistics}

The top 10 users who are responsible for the most tweets in te reo Māori are listed in Table 3.

Table 3: Most tweets in Māori

\begin{tabular}{|l|r|r|r|r|}
\hline User & $\begin{array}{r}\text { Māori } \\
\text { tweets }\end{array}$ & $\begin{array}{c}\text { Total } \\
\text { Tweets }\end{array}$ & $\begin{array}{c}\% \text { in } \\
\text { Māori }\end{array}$ & Followers \\
\hline maonewt & 29810 & 32020 & $93.1 \%$ & 21 \\
\hline maoold & 22390 & 24659 & $90.8 \%$ & 17 \\
\hline maobbl & 11620 & 57703 & $92.9 \%$ & 12 \\
\hline HURIMOZ & 7098 & 120002 & $5.9 \%$ & 1115 \\
\hline temihinga & 860 & 1232 & $69.8 \%$ & 297 \\
\hline waateanews & 746 & 8238 & $9.1 \%$ & 1414 \\
\hline TeKarereTVNZ & 681 & 2729 & $25.0 \%$ & 1306 \\
\hline MaoriStream & 658 & 18935 & $3.5 \%$ & 547 \\
\hline CmmdrZed & 554 & 18103 & $3.1 \%$ & 270 \\
\hline RatuTibble & 539 & 3155 & $17.1 \%$ & 218 \\
\hline
\end{tabular}

These users tweet for quite different reasons, as is explained below.

3.1.1. Religious Based Tweeters. The top three tweeters of Māori are religious based and make up $71 \%$ of te reo Māori tweets. The top two appear to have posted Māori translations of Bible script from the New Testament (maonewt) and from the Old Testament (maoold) and have posted $58 \%$ of Māori tweets. Both accounts now have a suspended status. There have been no postings from 'maonewt' since May 2013 nor from 'maoold' since June 2012. The third highest tweeter, 'maobbl', has 12 followers and posts verse from the Māori translation of the Bible - 
often referred to as the 'Māori Bible'. These tweets appear to originate out of California, USA and nothing has been posted since June 2013.

The top tweeter in Māori, 'maonewt', is credited with one third of the tweets in Māori. The highest user for Gàidhlig at 29\%, 'sconewt', also posts translations of the New Testament of the Bible in Scottish. Similarly, the top user for Cymraeg provides New Testament translations for Welsh. One might question the contribution that such religious-based postings provide to online language communities. Given the case for Māori, where the postings appear to originate more as part of a global offshore campaign, it might appear that the message is more important than the language, suggesting that the primary aim is dissemination rather than any motivation to promote minority languages. On the other hand, considering that the Cymraeg user 'newyddcymraeg' posts scripture in Welsh and has over 2000 followers, investigation aiming to understand the effect of these posts on language communities for the different cultures might be beneficial.

3.1.2. HURIMOZ. The fourth highest number of tweets in te reo Māori is from the user 'HURIMOZ'. This user provides nearly $8 \%$ of Māori tweets and is linked to a linguistic website; Huri Translations (http://www.huri-translations.pf/).

Huri Translations is based in Mo'orea, French Polynesia and is a consulting agency created in 2008 to provide language solutions and cultural knowledge to their clients. According to the website, this consultancy helps business owners localize their products and services using French Polynesian imagery and linguistics as a source of branding and marketing inspiration. This is done by "combining marketing techniques with syntaxic, semantic and pragmatic rules, the agency designs corporate identity - called branding - to meet naming projects and create logos, taglines etc.

Huri Translations is a multilingual website that is available in eight languages. The pages are primarily non-interactive and feature a strong sense of French Polynesian imagery and themes - even in the nonPolynesian languages. The website advertises services such as translation, localisation, transcription and proof-reading. They are also willing to engage in conversation regarding the development and marketing of customised branding and they boast a selection of 'language promotion tools' that includes a Polynesian keyboard for the simple provision of a tohutō (macron) when using Windows or Android OS. Users are able to view this website in te reo Māori.

The bulk of the 'HURIMOZ' tweets are in English and appear to focus mainly on regurgitating news items and retweeting topics of interest e.g. restaurants identified by other tweeters. Even so, nearly $6 \%$ of the 'HURIMOZ' total are tweets in te reo Māori, equating to nearly 7100 tweets. As mentioned earlier however, the posts in te reo Māori are largely commentary and don't appear to invite interaction amongst other users.

3.1.3. temihinga. The fifth highest number of tweets in te reo Māori is from the user profile 'temihinga'. User 'temihinga' is a facilitator for He Pikinga Reo, a program for the development of Māori-language teachers, with CORE Education. Based in Christchurch, New Zealand, CORE Education describes itself as “... a not-for-profit education consultancy, professional learning and research agency" [13]. According to their website they believe that new technologies are the most exciting way to engage 21st century learners across all education and training sectors.

With a history in the promotion of te reo Māori and the development of language speakers, 'temihinga' has a solid background with the education sector of New Zealand. This includes teaching in Māori-medium and mainstream schools, lecturing and tutoring at university and polytechnic level, and facilitating for various Māori-language strategies in the New Zealand. At 68.8\% Māori (see Table 3), the te reo ratio of 'temihinga' is relatively high and tweets appear to invite (and attract) interaction from other users in te reo Māori.

3.1.4. waateanews. The posts from 'waateanews' originate from Radio Waatea. Radio Waatea is an urban Māori radio and news station based in Auckland, New Zealand.

Most of the tweets are in English but this user has a relatively high number of followers. The postings are generally news items or commentary regarding items of news that are topical at the time.

3.1.5. TeKarereTVNZ. Te Karere is Television New Zealand's (TVNZ) news and current affairs programme that broadcasts entirely in the Māori language. The focus of the programme is content which is of some significance to the targeted Māori audience. As with waateanews, the tweets from TeKarereTVNZ consist of or are about topical news and current affairs. Given that the program broadcasts entirely in te reo Māori, it is interesting to note that only $25 \%$ of their tweets (see Table 3 ) are in te reo Māori.

\subsection{Other Significant Māori Tweeters}

In an effort to identify users who might have a more significant impact in terms of an online Māorilanguage community, the tweets have been filtered to include those users who have posted more than 50 tweets in te reo Māori and have more than a $50 \%$ te reo Māori content. Further, to distinguish those who 
might already affect other users an additional filter was added to detect those who have more than 50 followers. Only five users were identified that met all these criteria; they are displayed in Table 4.

Table 4: Profiles with $>50$ tweets $\&>50 \%$ in Māori $\&>50$ followers

\begin{tabular}{|l|r|r|r|r|}
\hline User & \multicolumn{1}{|c|}{$\begin{array}{c}\text { Māori } \\
\text { tweets }\end{array}$} & $\begin{array}{c}\text { Total } \\
\text { Tweets }\end{array}$ & $\begin{array}{c}\text { \% in } \\
\text { Māori }\end{array}$ & Followers \\
\hline temihinga & 860 & 1232 & $69.8 \%$ & 297 \\
\hline $\begin{array}{l}\text { PukanaMaiO } \\
\text { Whatu }\end{array}$ & 317 & 553 & $57.3 \%$ & 223 \\
\hline MaataaWaka & 192 & 283 & $67.8 \%$ & 127 \\
\hline hpr2013 & 79 & 100 & $79.0 \%$ & 159 \\
\hline Tiiwaha & 67 & 77 & $87.0 \%$ & 51 \\
\hline
\end{tabular}

Although the filter criteria for these profiles was chosen somewhat arbitrarily it does identify a small number of users who are tweeting in Māori and can serve as role models. The first profile, temihinga, has already been discussed above. The others will be examined in further detail below.

3.2.1. PukanaMaiOWhatu. Similar to TeKarere, tweets from user 'PukanaMaiOWhatu' are the offspring of Pūkana, a programme aired by New Zealand's Māori Television. This award-winning show is in its $15^{\text {th }}$ year of production and is described as colourful, hip and funny [14]. The show contains music, cheeky send-ups and practical life skills and is aimed at younger, pre-dominantly Māori, audiences.

The focus of Pūkana is content which is aimed at and engages the targeted, younger Māori audience. Recent tweets contain a large amount of competition conversation. Interestingly, much of the interaction generated by these tweets occurs as competition feedback from other followers within the Facebook pages included as links in the tweets. This feedback includes a high level of Instagram connections.

3.2.2. MaataaWaka. The posts from MaataaWaka originate from a Hauora (Māori Health Organisation) called Maataa Waka which is based in the northern area of New Zealand's South Island. This organisation has a vision of caring for people and their mission is "to provide tools within a Kaupapa Māori (Māori ideology) framework to make a positive difference for whānau (families) [15].

The services offered by Maata Waka are free of charge and appear to focus on strengthening child, family and community health. According to the services outlined by their website, they also offer Youth Services, Social Services and initiatives based around physical activity and nutrition.
3.2.3. hpr2013. User hpr 2013 arises from $\mathrm{He}$ Pikinga Reo, a Māori-language Teacher Development Program facilitated by Core Education based in Christchurch, New Zealand. The postings appear to be the work of one of the kaiako (teachers) whose own profile (wawaro81) is linked to hpr2013.

Of the 32 tweets posted by wawaro 81 herself, 18 $(56.2 \%)$ were in te reo Māori and she also has 73 followers.

3.2.4. Tiiwaha. Tiiwaha arose from one of many initiatives implemented in 2012 by a group at Massey University in the Manawatu region of New Zealand. As the nation celebrated Te Wiki o Te Reo Māori (Māori Language Week), during July 2012, this group facilitated a series of events in celebration of Te Marama o te Reo (Mãori Language Month) for the whole month. The original event, dubbed tiwwaha (speak up), was an informal Māori language mentoring group for staff and students run by a group of university staff. One of the group, Dr Darryn Joseph says tīwaha is not just for fluent speakers. "It's for anyone who wants to improve or use their Māori language skills in a supportive environment, outside of a classroom setting." [16].

An analysis of the Tiiwaha tweets shows that the majority of the tweets (77\%) occurred during Te Marama o te Reo (July 2012). The only posts in 2013 have been two August and one in January. Also, of the 77 tweets, 21 were retweets, where the tweets of other users have been re-posted by Tiiwaha.

\subsection{Low Percentage Māori Tweeters}

Despite the user profiles discussed above, in general the statistics for te reo Māori tweeters show that those that post the most tweets overall actually post very few in te reo Māori. In fact, from the 26 users who have posted over 10,000 tweets, excluding the four tweeters who have the highest percentage of Māori-language tweets from this group (See Table 3) shows that the remaining 22 users tweet in Māori less than $3 \%$ of the time.

These percentages may be misleading though. In the case of one particular tweeter, 'Kase_Marbles', $0.4 \%$ of their total tweets is still 434 tweets in Māori - almost double the calculated average per user. With over 3,700 followers and high levels of interaction with other tweeters, at least in English, $0.4 \%$ may yet have some influence on an online Māori-language community.

It may also be useful to identify tweets in terms of numbers and timespans but this has been difficult to determine from the Twitter website. Direct contact with selected users might prove informative. Additionally, users with relatively high numbers of followers could prove invaluable in efforts to build language communities in this medium. 


\subsection{Followers of Māori Tweeters}

When the user profiles are ranked by the number of followers, it is appears that the bulk of the followers of the higher ranked users are following the higher volume of non-Māori tweets. Users who tweeted in te reo Māori with the most number of followers are displayed in Table 5.

Table 5: Profiles with highest number of followers

\begin{tabular}{|l|r|r|r|r|}
\hline User & $\begin{array}{r}\text { Māori } \\
\text { tweets }\end{array}$ & $\begin{array}{r}\text { Total } \\
\text { Tweets }\end{array}$ & $\begin{array}{c}\text { \% in } \\
\text { Māori }\end{array}$ & Followers \\
\hline Te_Papa & 4 & 3183 & $0.1 \%$ & 10668 \\
\hline MaiFM & 5 & 3003 & $0.2 \%$ & 8417 \\
\hline nzbirds & 15 & 12643 & $0.1 \%$ & 5505 \\
\hline kingkapisi & 114 & 17270 & $0.7 \%$ & 4814 \\
\hline maoritv & 201 & 4792 & $4.2 \%$ & 4781 \\
\hline coreeducation & 24 & 3033 & $0.8 \%$ & 4558 \\
\hline tauhenare & 223 & 22544 & $1.0 \%$ & 3955 \\
\hline
\end{tabular}

Using the profile with the most followers, Te_Papa, as an example. Te_Papa has 10,668 followers but only $0.1 \%$ tweets in te reo Māori. The tweets arise from New Zealand's 'National Museum' - Te Papa and most of the postings refer to exhibitions and events facilitated by the museum. Given that most of Te_Papa's tweets are in English it appears that the high number of followers are more interested in the museum's information updates rather than any posting in te reo Māori.

The user profile with the $2^{\text {nd }}$ most followers is MaiFM with 8.417 followers, followed by nzbirds with 5,505 users. However these users have $0.1 \%$ and $0.2 \%$ respectively of tweets that are posted in te reo Māori. Again this suggests that the high number of followers are more interested in the non-Māori language tweets.

\section{Analysis and Discussion}

An analysis of the Indigenous Tweets website has shown that tweeting is occurring for indigenous languages and the number of languages and the number of tweets is increasing over time. It would be interesting to undertake some longitudinal studies to see if the number of tweets subsided after the initial excitement/honeymoon period - however this was beyond the scope of this paper.

What was apparent though was that in some languages a small amount of users were responsible for a disproportionally high number of tweets. This could indicate the potential that exists for some language communities in this form of social media. It shows that technically the ability to tweet is available in (many) indigenous languages. What is still needed is perhaps awareness and encouragement to utilise this form of social media by indigenous language activists and indigenous language speakers.

The further analysis of tweeting in te reo Māori has identified some important behaviours. The three most prolific tweeters of te reo Māori were disseminating scripts from the Bible, but had a low number of followers. Tweets from radio and television programmes, in particular Māori radio and Māori television programmes were also prominent. What was noted about these users was while they were high in one category (e.g. number of tweets) they were also low in another category (e.g. percentage of tweets in Māori, or number of followers).

In an effort to identify users of twitter that could have an impact in a language community a set of criteria were defined; more than 50 tweets, more than $50 \%$ of tweets in te reo Māori and more than 50 followers. This criteria identifies a more active, vibrant, communicating language group who, it could be suggested, were actively involved in using twitter to communicate in te reo Māori. For te reo Māori there were 5 profiles that matched this criteria; a Māori individual, a Māori television programme, a Māori health programme, a Māori language course, and a group supporting Māori language week. These profiles suggest that it is Māori that are seeking to support te reo Māori in the twitter sphere. However with 134,000 speakers of te reo Māori [17] the fact that only 5 profiles match this criteria is somewhat disconcerting.

Initial contact with two of the profiles who were identified as posting more than 50 tweets of which more than half were in te reo Māori and with more than 50 followers, has proven useful and positive. The users are kaiako (teachers/facilitators) with He Pikinga Reo described earlier as a language-teacher development program. Both kaiako say their goal is "... to get the reo out there" and they aim to increase the quality and quantity of the use of te reo Māori in this medium. Although there is an expectation from their organisation to promote te reo using whatever technology is available and suitable, the kaiako say they are often thrilled to be conversing with people in this medium that they have never met before in person $[18,19]$.

It is unclear what effect diaspora and wider spread communities has on the percentage uptake in Twitter since it is difficult to determine where people are tweeting from. Using Twitter in an indigenous language has created one environment where people are able to engage with others in their own language whether they are in the same neighbourhood or countries and continents apart. It would therefore seem ideal to understand what some of the key drivers are that would encourage people to engage within this medium. Some consideration should also be given to the 'spread of ethnicities' and how social 
media such as Twitter is or could be used to enable speakers of minority languages to stay in contact and speak with each other in their language from whereever they are in the world.

Further investigation to analyse differences between fostering conversation and disseminating translated text in minority languages online may prove useful. Firstly, to determine whether or not language health might somehow be supported or nurtured in both scenarios and secondly, to identify how each scenario might contribute to doing so.

Further comparative studies between selected languages would identify issues that are faced by most minority language users but may also highlight nuances that are more culture-specific. Understanding these issues is important on a broader scale, but also can offer wisdoms to individual language communities that are turning to social media to assist in language survival strategies.

\section{Conclusions}

The purpose of this research was to determine if one particular form of social media, tweeting, could assist with the promotion and propagation and ultimately the revitalization of an indigenous language.

The research was made possible due to the indigenous tweeting data that is captured by the Indigenous Tweets website. This website currently captures tweeting data for over 150 indigenous languages - a statement within itself that confirms tweeting is being used in 150 indigenous languages. But how well is tweeting being used? And what impact is tweeting in an indigenous language having, especially in regards to language health?

The previous sections have shown that tweeting is being undertaken extensively by languages such as Euskara, Kreyòl Aylsyen and Cymraeg and having the ability to tweet has provided these languages with another opportunity to develop and flourish.

The case for te reo Māori is not so convincing. The profiles show that the primary purpose for many of the larger tweeters is for religious purposes or for the dissemination of notices and news items. While it could be argued that this still qualifies as language promotion, the conversations are unidirectional.

However it is evident that a small number are actively using twitter to converse in te reo Māori. Whether this small group multiplies and gets saturation in the Māori language community, or whether the enthusiasm for this medium fades away over time... is yet to be determined.

\section{References}

[1] Chrisp, S. (2005). Māori Intergenerational Language Transmission. International Journal of the Sociology of Language, 172,149-181.
[2] Dyson, L., \& Underwood, J. (2005). 'Indigenous People on the Web.' www.jtaer.com/apr2006/dyson _underwood_p6.pdf (17 October 2013).

[3] Ungerleider, N. (2011). 'Preserving Indigenous Languages Via Twitter.' www.fastcompany.com /1747283/indigenous-tweet-preserving-indigenouslanguages-via-twitter (17 October 2013).

[4] Avila, E. (2011). 'Twitter to Promote and Preserve Under-represented Languages.' http://rising.globalvoicesonline.org/blog/2011/07/18/twitte r-to-promote-and-preserve-underrepresentedlanguages/print/ (17 October 2013).

[5] Fishman, J. (1991). Reversing language shift: Theoretical and empirical foundations of assistance to threatened languages. Philadelphia, USA: Multilingual Matters Ltd.

[6] Scannell, K. (2011). 'Indigenous Tweets - Welcome.' http://indigenoustweets.blogspot.co.nz/ 2011/03/welcomefailte.html (17 October 2013).

[7] Scannell, K. (2011b). 'How many languages are out there?' http://indigenoustweets.blogspot .co.nz/2011/03/how-many-languages-are-out-there.html.

(17 October 2013).

[8] Personal Communication; Kevin Scannell to Paora Mato. (23 October 2013).

[9] Scannell, K. (2011c). '1000 languages on the Web.' http://indigenoustweets.blogspot.co.nz/ 2011/12/1000languages-on-web.html (17 October 2013).

[10] Lee, D. (2011). 'Micro-blogging in a mother tongue on Twitter.' http://news.bbc.co.uk/2/hi /programmes/click_ online/9450488.stm (17 October 2013).

[11] Omniglot. (2013). 'The online encyclopedia of writing systems and languages: Haitian Creole.' http://www.omniglot.com/writing/haitiancreole.htm (17 October 2013).

[12] BBC News Wales. (2012). 'Census 2011: Number of Welsh speakers falling.' http://www.bbc.co.uk/news/ukwales-20677528 (17 October 2013).

[13] Core Education. (2013). 'Welcome to Core Education.’ http://www.core-ed.org/ (17 October 2013).

[14] Māori Television. (2013). 'Pūkana.' http://www.maoritelevision.com/tv/shows/pukana (17 October 2013).

[15] Maataa Waka Ki Te Tau Ihu Trust. (2013). 'Maataa Waka Ki Te Tau Ihu Trust.' www.maataawaka.co.nz/ (17 October 2013).

[16] Massey University. (2012). 'Māori language month at Massey' http://www.massey.ac.nz/massey/aboutmassey/news/article.cfm?mnarticle_uuid=F42E09CDDAAA-6AFC-E262-80766DFA2764 (17 October 2013). 
[17] Waitangi Tribunal. (2010). Pre-publication, WAI 262 Te Reo Māori. Wellington: Waitangi Tribunal.

[18] Personal Communication; Wawaro Te Whaiti to Paora Mato. (31 October 2013).

[19] Personal Communication; Te Mihinga Komene to Paora Mato \& Te Taka Keegan. (4 November 2013).

\section{Acknowledgements}

We acknowledge Professor Kevin Scannell for his work in the creation of the Indigenous Tweets website, the generous sharing of the data contained within and his candid feedback to our questions and queries.

We also acknowledge Ngā Pae o te Māramatanga, based at the University of Auckland, New Zealand, for their fantastic support of this research by way of financial doctoral scholarship and the provision of the Ngā Pae o te Māramatanga Conference Attendance Grants which enabled us to present this research at the Canada International Conference for Education 2013 in Toronto, Canada.

This research has also attracted generous and much-appreciated financial support from the Tūwharetoa Māori Trust Board, the Tūwharetoa Settlement Trust, the Lake Taupo Forest Trust, the Office of the Pro Vice-Chancellor Māori and the School of Computer Science of the University of Waikato, New Zealand. 